\title{
Evaluating Bone Mineral Density in Pediatric Acute Lymphoblastic Leukemia Survivors: A Tertiary Care Hospital Experience
}

\author{
Ersin Toret $^{1 *}$, Burcu Dural ${ }^{1}$, Yeter Duzenli Kar ${ }^{1}$, Zeynep Canan Ozdemir ${ }^{1}$, Ilknur Ak Sivrikoz and Ozcan \\ Bor $^{1}$ \\ ${ }^{1}$ Department of Pediatric Hematology-Oncology, Faculty of Medicine, Eskisehir Osmangazi University, \\ Eskisehir, Turkey \\ ${ }^{2}$ Department of Nuclear Medicine, Faculty of Medicine, Eskisehir Osmangazi University, Eskisehir, Turkey

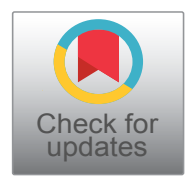

*Corresponding author: Ersin Toret, MD, Department of Pediatric Hematology-Oncology, Faculty of Medicine, Eskisehir Osmangazi University, 26100, Eskisehir, Turkey, Tel: +90-505-799-4234

\begin{abstract}
Acute leukemias are the most common malignancies seen in children and they account for one third of childhood cancers. Children who survived ALL, experience reduced bone mineral density (BMD) due to the disease, long-lasting glucocorticoid usage, chemotherapy toxicities, nutritional deficiencies and physical immobility. The decreased BMD have determined at all stages of disease. This retrospective cross-sectional study purposes to analyse the results of BMD in children who have completed their ALL treatment. Data of 58 pediatric ALL survivors whose z-scores were calculated by DEXA, were reviewed from their medical records. Twenty-one (38\%) patients had normal BMD. Low bone mineral density was detected in $29(50 \%)$ and osteoporosis was detected in $8(12 \%)$ patients. There was nostatistically significant difference between BMD and sex, immunophenotype, leukemia risk group or age at diagnosis $(p>0.05)$. Only, a significant difference was found between BMD and vitamin D supplementation received or not received during chemotherapy $(p<0.05)$. Also There was no statistically significant difference between mean levels of $\mathrm{Ca}, \mathrm{P}, \mathrm{ALP}$, vitamin $\mathrm{D}$ and result of DEXA $(p>0.05)$. Children with cancers such as ALL are malnourished and have limited physical activity during under chemotherapy. Nutrition and physical activity supporting a growing healthly bone should be provided for all children with cancer from the start of chemotherapy until bone gowth is complete.
\end{abstract}

\section{Keywords}

Acute lymphoblastic leukemia, Bone mineral density, Children, Osteoporosis, Survivors

\section{Introduction}

Acute leukemias are the most common malignancies seen in children and they account for one third of childhood cancers. They are occurred abnormal proliferation of hematopoietic stem cells leading to interruption of normal healthy cells production. The survival rate for acute leukemia has rised as a result of more intensive chemotherapy, advanced supportive care and better followed-up conditions. Acute lymphoblastic leukemia (ALL) is the most common group with the best results achieved $[1,2]$. However, children who survived ALL, experience reduced bone mineral density (BMD) due to the disease, long-lasting glucocorticoid usage, chemotherapy toxicities, nutritional deficiencies and physical immobility. The decreased BMD have determined at all stages of disease. Also avascular necrosis is an another annoying musculoskeletal complication of pediatric leukemia survivors. Particularly both osteoporosis and osteonecrosis are occured as a result of glucocorticoid treatment [3-5]. Dual-energy X-ray absorptiometry (DEXA) is an one of a conventional method for evaluating BMD [4]. In this study, we decided to analyse the results of $B M D$ in children who have completed their ALL treatment.

\section{Materials and Methods}

This retrospective cross-sectional study was performed at a tertiary care hospital in Turkey. The study 
was approved by the locale ethics committee. Throughout 2013 to 2020, data of 58 pediatric ALL survivors whose z-scores were calculated by DEXA, were reviewed from their medical records. Demographic features of patients were enrolled. Z-score values above -1 were accepted as normal, between -1 to -2 as low bone density and below -2 as osteoporosis according to the defenition of International Society of Clinical Densitometry [6]. DEXA results were assessed from the lumbarspine (L1 to L4).

All patients were classified to risk groups and treated with the ALL intercontinental berlin-frankfurt-münster (ALL-IC BFM) 2009 protocol. The risk group of patients were defined as standart (SRG), intermediate (IRG) or high (HRG) according to age, white blood cell (WBC) count at diagnosis, day eight responce to prednisone, immunophenotype and some prognostically precisely proven genetic markers such as $t(9 ; 22), t(4 ; 11)$ and/ or their moleculer equvalents $B C R / A B L$ and MLL/AF4, respectively. The ALL-IC BFM 2009 protocol includes high dose prednisone for a month during the induction therapy, in addition, there induction phase consists of high dose dexamethasone for three weeks for all risk groups. The treatment lasts two years in the ALL-IC BFM 2009 protocol. Evaluations including DEXA are made at the latest $27^{\text {th }}$ month of leukemia diagnosis or within the first three months after cessation of treatment. Patients who were underwent hematopoietic stem cell transplantation, were treated with radiotherapy or had other disease or non-leukemic predisposing factors for low bone denstiy, were excluded. Collected data were analyse dusing descriptive statistics; frequency, mean and standard deviation were calculated. Mann Whitney $\mathrm{U}$ test and Krukal Wallis test were used to analyse BMD and features of patients. One Way Anova test was used to compare mean level of calcium, phosphorus, alkaline phosphatase and vitamin $D$ according to result of DEXA. A $p$-value $<0.05$ was considered significant.

\section{Results}

Fifty-eight patients ( 26 males, 32 females) were participated to this study. Table 1 shows the patients' demographic characteristics. The meanage was $5.5 \pm 3.8$, the median age was 4.2 year (interquartile range 4.3). Patients were $17(30 \%)$ in SRG, $36(61 \%)$ in IRG and 5 (9\%) in HRG. The immunophenotype distribution was determined as pre-B ALL in 50 (86\%) and pro-B ALL in eight (14\%) patients. Twenty-three (40\%) patients received vitamin D supplementation under chemotherapy due to their vitamin $D$ levels were determined lower than $20 \mathrm{ng} / \mathrm{ml}$. The mean and median BMD were determined as $0.52 \pm 0.13$ and $0.48 \mathrm{~g} / \mathrm{cm}^{2}$ (interquartile range $0.11)$, respectively. Additionally, the mean and median Z-score were calculated as-1.82 \pm 3.75 and -1.5 (interquartile range -1.4$)$. Twenty-one (38\%) patients had normal BMD. Low bone mineral density was detected in $29(50 \%)$ and osteoporosis was detected in $8(12 \%)$
Table 1: Characteristics of patients.

\begin{tabular}{|c|c|c|}
\hline Variable & $\mathbf{n}$ & $\%$ \\
\hline \multicolumn{3}{|l|}{ Sex } \\
\hline Male & 26 & 45 \\
\hline Female & 32 & 55 \\
\hline \multicolumn{3}{|c|}{ Age at diagnosis (year) } \\
\hline Mean \pm SD & $5.5 \pm 3.8$ & \\
\hline Median $\left(\mathrm{IQR}_{25-75}\right)$ & $4.2(4.3)$ & \\
\hline \multicolumn{3}{|l|}{ ALL risk group } \\
\hline SRG & 17 & 30 \\
\hline IRG & 36 & 61 \\
\hline HRG & 5 & 9 \\
\hline \multicolumn{3}{|c|}{ Immunophenotypes } \\
\hline Pre-B & 50 & 86 \\
\hline Pro-B & 8 & 14 \\
\hline T-cell & - & - \\
\hline \multicolumn{3}{|c|}{$\begin{array}{l}\text { Vitamin D supplementation } \\
\text { while under chemotherapy }\end{array}$} \\
\hline Received & 23 & 40 \\
\hline Not received & 25 & 60 \\
\hline \multicolumn{3}{|c|}{ Bone mineral density $\left(\mathrm{g} / \mathrm{cm}^{2}\right)$} \\
\hline Mean \pm SD & $0.52 \pm 0.13$ & \\
\hline Median $\left(\mathrm{IQR}_{25-75}\right)$ & $0.48(0.11)$ & \\
\hline \multicolumn{3}{|l|}{ Z-score } \\
\hline Mean \pm SD & $-1.82 \pm 3.75$ & \\
\hline Median $\left(\mathrm{IQR}_{25-75}\right)$ & $-1.5(-1.4)$ & \\
\hline \multicolumn{3}{|c|}{ BMD according to z-score } \\
\hline Normal & 21 & 38 \\
\hline Low bone density & 29 & 50 \\
\hline Osteoporosis & 8 & 12 \\
\hline
\end{tabular}

SD: Standard deviasion; IQR: Interquartile range; ALL: Acute lymphoblastic leukemia; SRG: Standard risk group; IRG: Intermediate risk group; HRG: High risk group; BMD: Bone mineral density

patients. With regard to Table 2; there was no statistically significant difference between BMD and sex, immunophenotype, leukemia risk group or age at diagnosis. Only, a significant difference was found between $\mathrm{BMD}$ and vitamin $\mathrm{D}$ supplementation received or not received during chemotherapy. In patients who received vitamin $\mathrm{D}$ during chemotherapy, the BMD was normal in 17, low bone density in 15 and osteoporosis in two patients. Further more in patients who did not receive vitamin $\mathrm{D}$ during chemotherapy, the BMD was normal in four, low bone density in 14 and osteoporosis in six patients. The means of vitamin $D$, calcium $(\mathrm{Ca})$, phosphorus (P) and alkaline-phosphatase (ALP) levels at similar times to DEXA are shown in Table 3. There was no statistically significant difference between mean levels of Ca, P, ALP, vitamin D and result of DEXA.

\section{Discussion}

The overall survival of children with ALL has been 
Table 2: Comparison of factors that affect the BMD.

\begin{tabular}{|c|c|c|c|c|c|c|c|c|c|c|c|}
\hline & \multicolumn{2}{|c|}{$\operatorname{Sex}(n)$} & \multicolumn{2}{|c|}{$\begin{array}{l}\text { Immunophenotype } \\
\text { (n) }\end{array}$} & \multicolumn{3}{|c|}{ ALL Risk Group (n) } & \multicolumn{2}{|c|}{$\begin{array}{l}\text { Age at diagnosis } \\
\text { (n) }\end{array}$} & \multicolumn{2}{|c|}{ Vitamin D supp. $^{* * *}$} \\
\hline & Male & Female & Pro-B & Pre-B & SRG & IRG & HRG & $<7$ years & $\begin{array}{l}\geq 7 \\
\text { years }\end{array}$ & Received & $\begin{array}{l}\text { Not } \\
\text { received }\end{array}$ \\
\hline Normal & 6 & 15 & 3 & 18 & 9 & 10 & 3 & 15 & 6 & 17 & 4 \\
\hline $\begin{array}{l}\text { Low bone } \\
\text { density }\end{array}$ & 15 & 14 & 3 & 26 & 8 & 19 & 2 & 22 & 7 & 15 & 14 \\
\hline Osteoporosis & 5 & 3 & 2 & 6 & 1 & 6 & 0 & 5 & 3 & 2 & 6 \\
\hline$P$ value & \multicolumn{2}{|c|}{$0.055^{*}$} & \multicolumn{2}{|l|}{$0.728^{*}$} & \multicolumn{3}{|c|}{$0.149^{* *}$} & \multicolumn{2}{|l|}{$0.856^{*}$} & \multicolumn{2}{|l|}{$0.004^{*}$} \\
\hline
\end{tabular}

ALL: Acute lymphoblastic leukemia; SRG: Standard risk group; IRG: Intermediate risk group; HRG: High risk group; "Mann-whitney U test; " Kruskal wallis test; " Vitamin D supplementation while under chemotherapy, $p$ of significance if $<0.05$.

Table 3: Serum mean level of calcium, phosphorus, alkaline phosphatase and vitamin D at similar times to DEXA according to BMD.

\begin{tabular}{|c|c|c|c|c|}
\hline BMD & Calcium ( \pm SD) & Phosphorus ( \pm SD) & Alkaline phosphatase ( \pm SD) & Vitamin D ( \pm SD) \\
\hline Normal & $9.8 \pm 0.3$ & $4.7 \pm 0.5$ & $317 \pm 188$ & $21.8 \pm 8.5$ \\
\hline Low bone mineral density & $9.7 \pm 0.5$ & $4.8 \pm 0.5$ & $311 \pm 201$ & $24.2 \pm 9.8$ \\
\hline Osteoporosis & $9.7 \pm 0.4$ & $5.1 \pm 0.4$ & $280 \pm 168$ & $27.2 \pm 6.4$ \\
\hline$P$ value ${ }^{*}$ & 0.787 & 0.167 & 0.920 & 0.434 \\
\hline
\end{tabular}

BMD: Bone mineral density; DEXA: Dual-energy X-ray absorptiometry; SD: Standard deviasion; *One way Anova test

increasing over the years through to multidisciplinary cooperation grounded to chemotherapy and advanced supportive therapies. Despite increasing survival, longterm complications such as bone mineralization defects are observed in thriving children with ALL due to osteotoxic effects of leukemia treatment, decreased physical activity and malnutrition [7-9]. A diet rich or supplemental calcium and vitamin $D$ intake have recommended for patients during chemotherapy [3,4]. Gunes, et al. [9] reported that low calcium and vitamin $D$ intake could have provided to an abnormal bone mineralization in patients undergoing treatment of ALL and they recommend prophylactic vitamin $D$ to patients in the first two years of treatment which was found the most critical period for BMD decrease [9]. In contrast with this study, Diaz, et al. [10] and Demirsoy, et al. [11] reports found that there was not any significant between the calcium-vitamin D supplementation and BMD.

Skeletal abnormalities such as low BMD, osteoporosis and osteonecrosis are common seen in pediatric ALL survivors. While BMD is progressively decreased in children who survived from leukemia, anthropometric parameters recover in the long-term [12]. Donmez, et al. [13] found osteopenia in $23.1 \%$ and osteoporosis in $7.7 \%$ of patients in their survey. In another study from Turkey, reported low BMD in $32 \%$, osteoporosis in $24 \%$ and osteonecrosis in $16 \%$ of patients [14]. We found low BMD in $50 \%$ and osteoporosis in $12 \%$ of patients in our study. Considering that race, climate and dietary habits also affect BMD, we found similar results when we compared similar studies from our country. When the factors affecting to BMD are reviewed, Inaba, et al. [3] reported that there was statistically significant difference between age, leukemia risk group and BMD at week 120 from diagnosis. Under 10 year age or standard/high risk patients had higher risk to having lower BMD than over 10 year age or low risk patients. Also there was no significant difference between sex, white blood cell (WBC) count at diagnosis, cell lineage, central nervous system (CNS) involvement and BMD. In another study, significant differences were found between patients who completed treatment more than two years ago, daily calcium intakes, decreased physical activity and BMD [9]. In Donmez, et al. [13] study, they reported that there was no significant difference between leukemia risk group, pubertal status, passing time from cessation of treatment to DEXA and BMD. Vitanza, et al. [15] found that the overall difference in BMD after six years from cessation of chemotherapy among three age groups patients defined as ages under five years group 1 , ages five to 10 years group 2 and ages above 10 years at the time of diagnosis, significant difference was found between group 1 and group 3 . Similar to Vitanza, et al. [15] study, Erdem, et al. [14] found that the rate of osteonecrosis and bone changes were significantly higher in patients aged above 10 years. As summarized above, varying results were obtained on factors affecting to BMD in different studies. In our study, among factors affecting to BMD, only vitamin $D$ supplementation was found statistically significant while chemotherapy was ongoing. However, we supported patients who were diagnosed with vitamin D deficiency, In another respect, bone mineral abnormality was detected in $17(50 \%)$ patients who received vitamin $D$ and two of them were compatible with osteoporosis.

Gunes, et al. [9] recommended vitamin D prophylaxis to pediatric ALL patients as long as chemotherapy continues. Additonally, physical activity in addition to 
calcium and vitamin D supplementation are important to preventing insufficient bone mineralization in pediatric ALL survivors $[8,16]$. The bone biomarkers such as serum Ca, P, ALP and vitamin D levels were not different among patients and there was no significant difference between bone biomarkers and BMD $[9,12]$. Also the mean levels vitamin $D$ and insulin-like growth factor (IGF-1) were lower in patients than controls, there was no correlation between BMD and lower levels of those. In our study, there was no significant difference between bone biomarkers and bone changes.

In conclusion, now the goal is to prevent pediatric ALL survivors from long-term side effects which may adversely affect quality of life. The bone changes can cause skeletal morbidities, life-long pain and disabilities. A growing skeleton needs nutritional $\mathrm{Ca}, \mathrm{P}$, magnesium, vitamin $D$ and of course physical activity. Children with cancers such as ALL are malnourished and have limited physical activity during under chemotherapy.

Nutrition and physical activity supporting a growing healthly bone should be provided for all children with cancer from the start of chemotherapy until bone gowth is complete. Vitamin D levels and bone biomarkers should be checked intermitently in children with acute leukemia or other cancers during chemotherapy. Vitamin D or mineral supplementation should be given to patients who were shown deficiencies.

\section{Compliance and Ethical Standarts}

\section{Conflict of interest}

The authors declare that they have no conflict of interest.

\section{Ethical approval}

All procedures performed in studies involving human participants were in accordance with the ethical standards of the National Cancer Institute research committee and with the 1964 Helsinki declaration and its later amendments or comparable ethical standards.

\section{References}

1. Ward E, De Santis C, Robbins A, Kohler B, Jemal A (2014) Childhood and adolescent cancer statistics. CA Cancer J Clin 64: 83-103.

2. Seth R, Singh A (2015) Leukemias in children. Indian J Pediatr 82: 817-824.

3. Inaba H, Cao X, Han AQ, Panetta CJ, Ness KK, et al. (2018) Bone mineral density in children with acute lymphoblastic leukemia. Cancer 124: 1025-1035.
4. Ghassemi A, Banihashem A, Ghaemi N, Elmi S, Sayyar RE, et al. (2016) Evaluation of bone mineral density in children with acute lymphoblastic leukemia (ALL) and Non-hodgkin's lymphoma (NHL): Chemotherapy with/without Radiotherapy. Int J Hematol Oncol Stem Cell Res 10: 153-160.

5. Rohani F, Arjmandi RafsanjaniKh, Bahoush G, Sabzehparvar M, Ahmadi M (2017) Bone mineral density in survivors of childhood acute lymphoblastic leukemia. Asian Pac J Cancer Prev 18: 535-540.

6. Crabtree NJ, Arabi A, Bachrach LK, Fewtrell M, International society for clinical densitometry (2014) Dual-energy $\mathrm{x}$-ray absorptiometry interpretation and reporting in children and adolescents: The revised 2013 ISCD Pediatric Official Positions. J Clin Densitom 17: 225-242.

7. Pizzo PA, Poplack DG (2011) Principles of multimodal therapy" in Principles and practice of pediatric oncology, Lippincott, Philadelphia, USA.

8. Mostoufi-Moab S, Halton J (2014) Bone morbidity in childhood leukemia: Epidemiology, mechanisms, diagnosis, and treatment. Curr Osteoporos Rep 12: 300-312.

9. Gunes AM, Can E, Saglam H, Ilcol YO, Baytan B (2010) Assessment of bone mineral densityand risk factors in children completing treatment for acute lymphoblastic leukemia. J Pediatr Hematol Oncol 32: e102-e107.

10. Diaz PR, Neira LC, Fischer SG, Teresa Torres MC, Milinarsky AT, et al. (2008) Effect of 1,25(OH)2-vitamin D on bone mass in children with acute lymphoblastic leukemia. $J$ Pediatr Hematol Oncol 30: 15-19.

11. Demirsoy U, Sarper N, Aylan Gelen S, Zengin E, Kum T, et al. (2017) The association of oral vitamin d and calcium supplementation with bone mineral density in pediatric acute lymphoblastic leukemia patients. J Pediatr Hematol Oncol 39: 287-292.

12. Demirkaya M, Sevinir B, Saglam H (2011) Time-dependent alterations in growth and bone health parameters evaluated at different post treatment periods in pediatric oncology patients. Pediatr Hematol Oncol 28: 588-599.

13. Donmez AD, Isik P, Cetinkaya S, Yarali N (2019) Bone loss in pediatric survivors of acute lymphoblastic leukemia. Eurasian J Med 51: 38-41.

14. Erdem M, Tufekci O, Kızıldag S, Yılmaz S, Kızmazoglu $D$, et al. (2019) Investigation of the relationship between Fok1 and Col1A1 gene polymorphisms and development of treatment-related bone complications in children with acute lymphoblastic leukemia. Turk $\mathrm{J}$ Haematol 36: 12-18.

15. Vitanza NA, Hogan LE, Zhang G, Parker RI (2015) The progression of bone mineral density abnormalities after chemotherapy for childhood acute lymphoblastic leukemia. J Pediatr Hematol Oncol 37: 356-361.

16. Ahn MB, Suh BK (2020) Bone morbidity in pediatric acute lymphoblastic leukemia. Ann Pediatr Endocrinol Metab 25: 1-9. 